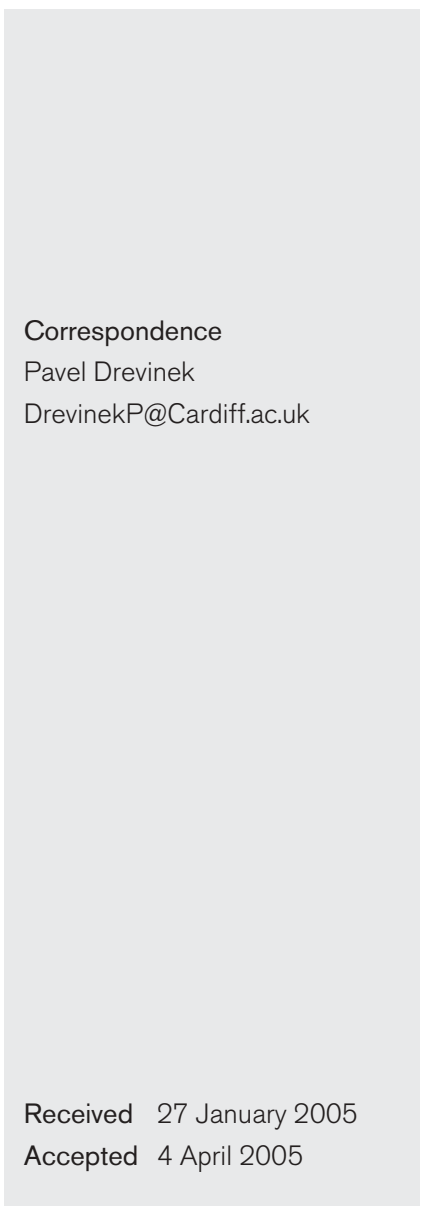

\title{
Widespread clone of Burkholderia cenocepacia in cystic fibrosis patients in the Czech Republic
}

\author{
Pavel Drevinek, ${ }^{1,2}$ Sarka Vosahlikova, ${ }^{1}$ Ondrej Cinek, ${ }^{1}$ Vera Vavrova, ${ }^{1}$ \\ Jana Bartosova, ${ }^{1}$ Petr Pohunek ${ }^{1}$ and Eshwar Mahenthiralingam ${ }^{2}$ \\ ${ }^{1}$ Paediatric Department, Charles University 2nd Medical School, Prague, Czech Republic \\ ${ }^{2}$ Cardiff School of Biosciences, Cardiff University, Main Building, Museum Avenue, PO Box 915, \\ Cardiff, Wales, UK
}

\begin{abstract}
The morbidity and mortality rates in patients with cystic fibrosis (CF) are significantly affected by infections with Burkholderia cepacia complex. In a Czech CF Centre, the prevalence of the infection reached up to $30 \%$, with the majority of patients found to be infected with Burkholderia cenocepacia (formerly genomovar III of the Burkholderia cepacia complex). Since B. cenocepacia is associated with patient-to-patient transmission and epidemic outbreaks among CF patients, this study sought to examine the epidemiological relatedness between the Czech isolates belonging to the genomovar-homogeneous group. Eighty-three clinical isolates recovered from $67 \mathrm{CF}$ patients were analysed using a random amplified polymorphic DNA (RAPD) assay and macrorestriction typing (Spel and Xbal) followed by PFGE. A single predominant banding pattern shared by multiple isolates was detected, although Spel-generated PFGE results yielded a higher rate of inter-pattern variability in comparison to the more uniform RAPD and Xbal-generated PFGE results for this clone. Both typing systems also showed that only three out of 67 patients harboured strains distinct from the major strain type. The dominant clone was characterized by PCR positivity for the B. cepacia epidemic strain marker, PCR negativity for the cable pilin subunit gene and close genetic relatedness to the epidemic strain of RAPD 01 type previously identified in Canada.
\end{abstract}

\section{INTRODUCTION}

Infections with micro-organisms from the Burkholderia cepacia complex (Bcc) are highly problematic for patients with cystic fibrosis (CF). These infections represent burdens not only for individual infected patients but also for the CF community as a whole since certain Bcc strains have the capacity of patient-to-patient transmission (Mahenthiralingam et al., 2002).

Most transmissible strains have been identified within the recently designated species Burkholderia cenocepacia (genomovar III of the Bcc) (Vandamme et al., 2003). For example, strains from the cable-pilus-encoding ET12 lineage have been responsible for epidemic outbreaks in a Toronto CF centre, and subsequently were spread to the UK (Johnson et al., 1994; Sun et al., 1995). Other transmissible strains within B. cenocepacia but distinct from the ET12 lineage have been identified in Canada [strain types designated RAPD 01, 04 and 06 (Speert et al., 2002); belonging to recA-derived subgroup IIIA] and in the USA [PHDC strain (Chen et al.,

Abbreviations: Bcc, Burkholderia cepacia complex; BCESM, B. cepacia epidemic strain marker; CF, cystic fibrosis; RAPD, random amplified polymorphic DNA.
2001) and Midwest clone (LiPuma et al., 1988); belonging to recA group IIIB]. Recently published data on PHDC and its presence in Europe indicate that transatlantic spread of the $\mathrm{Bcc}$ is not a feature exclusive to the ET12 lineage (Coenye et al., 2004).

The Czech CF community represents a CF population with high Bcc prevalence, reaching up to $30 \%$ during the late 1990s (P. Drevinek, unpublished data). Such a high figure may indicate that transmissible strains of the Bcc are present within the patient population. This assumption was further emphasized by assignment of more than $90 \%$ of isolates into the B. cenocepacia recA IIIA category (Drevinek et al., 2003). However, to reveal the true epidemic origin of the infection, further analysis of the genomovar-homogeneous groups was necessary.

In this study, we performed random amplified polymorphic DNA (RAPD) analysis (Mahenthiralingam et al., 1996) and macrorestriction typing followed by PFGE (Mahenthiralingam et al., 2001) to investigate genetic relatedness between Czech clinical isolates and to compare them with major epidemic strains from other CF centres. In addition, the isolates were also screened for the presence of genetic markers of strain transmissibility, i.e. the B. cepacia epidemic strain 
marker (BCESM) (Mahenthiralingam et al., 1997), a common genetic marker present in several well-described $B$. cenocepacia epidemic strains, and the cblA gene (Sajjan et al., 1995) specific for the ET12 strain.

\section{METHODS}

Patients and bacterial strains. Over the period 1997-2003, a total of 83 B. cenocepacia clinical isolates recovered from 67 CF patients had been stored. These 67 patients ( 34 males, 33 females; age 9-31 years) represented the majority (94\%) of Bcc-infected patients attending the Prague CF Centre. Single isolates were archived from 51 CF patients and two serial isolates recovered with a mean time interval of 38 months (range 22-54 months) were available from sixteen additional patients.

To check the accuracy of culture-based identification and to determine the genomovar status of the Bcc, extracted DNA from each isolate was subject to generic and genomovar-specific PCRs as described previously (Drevinek et al., 2002). All the isolates were assigned to B. cenocepacia, recA group IIIA. For further comparative analyses, the B. cenocepacia strains of several epidemic lineages were included in the analysis (Speert et al., 2002): C5635 and C6965 (RAPD type 01 strains), C5424 and J2315 (RAPD type 02, ET12 lineage), C5726 and C6433 (RAPD type 04), and C8182 and C7748 (RAPD type 06).

RAPD typing. For PCR-based typing, bacterial DNA was extracted from fresh overnight cultures using the QIAamp DNA Mini kit (Qiagen). RAPD assays were performed according to a previously described protocol (Mahenthiralingam et al., 1996) with slight modifications based on the use of a fluorescently tagged (Cy-5) primer 270 that enabled visualization of the amplification products on an ALFexpress II DNA sequence analyser (Pharmacia). The fragments were separated in 3.75\% Long Ranger gels (BMA) with $7 \mathrm{M}$ urea and $1 \times \mathrm{TBE}$ buffer $(\mathrm{pH} 8 \cdot 4)$ at $55^{\circ} \mathrm{C}$ and $1500 \mathrm{~V}$ for $10 \mathrm{~h}$. In-house-made PCR fragments of 800, 1100 and $1500 \mathrm{bp}$ in length were used as molecular size standards. Banding patterns were compared visually.

PFGE typing. Prior to processing for PFGE, bacterial cultures were grown in Tryptone soya broth with shaking (200 r.p.m.) at $37^{\circ} \mathrm{C}$ overnight. The density of harvested bacteria was adjusted to $\mathrm{OD}_{620} 0 \cdot 8-$ $0 \cdot 9$ and thereafter culture cells were embedded in plugs with low gelling temperature agarose type VII (Sigma). The plugs were lysed overnight with $1 \% N$-lauroyl sarcosine sodium salt and $0 \cdot 1 \%$ Pronase (Roche). Macrorestriction of genomic DNA was performed using either $3 \mathrm{U}$ of SpeI or $2.5 \mathrm{U}$ of $\mathrm{XbaI}$ enzymes at $37^{\circ} \mathrm{C}$ overnight. Fragments were separated on a $1.2 \%$ agarose gel with $0.5 \times \mathrm{TBE}$ running buffer at $14^{\circ} \mathrm{C}$ and $6 \mathrm{~V} \mathrm{~cm}{ }^{-1}$ on a CHEF-DR2 device (Bio-Rad). For SpeI-digested DNA, PFGE conditions were as follows: 1 to $40 \mathrm{~s}$ for $10 \mathrm{~h}$ and 30 to $90 \mathrm{~s}$ for $14 \mathrm{~h}$. XbaI-digested DNA was separated under the ramp conditions of 2 to $28 \mathrm{~s}$ for $20 \mathrm{~h}$. A lambda ladder was used as a standard size marker. Banding patterns were compared visually and defined criteria (Tenover et al., 1995) were used to determine the pulsotype of each isolate.

Epidemic strain-specific PCR. PCR assays targeting two markers of transmissibility, $c b l A$ and BCESM, were performed in accordance with published protocols (Sajjan et al., 1995; Mahenthiralingam et al., 1997). In addition, PCR detecting eubacterial $16 \mathrm{~S}$ rDNA sequence was included in each PCR reaction as an internal control of successful amplification (primers published by Mahenthiralingam et al., 2000).

\section{RESULTS AND DISCUSSION}

The high prevalence of $B$. cenocepacia-infected patients present in the Czech CF community was a cogent reason for performing active surveillance using genotyping meth- ods. To assess the genetic relationship between $B$. cenocepacia isolates, two widely used fingerprinting techniques were applied. RAPD typing was primarily chosen for its unquestionable advantage of ease of performance and its expected satisfactory discriminatory power when analysing large populations of micro-organisms collected over extended periods of time. A higher discriminatory power can be achieved by PFGE analysis, which also disposes excellent reproducibility with narrow inter-gel variability (Coenye et al., 2002; Tenover et al., 1995). Therefore, PFGE as a gold standard of bacteriological typing was used to check the propriety of the RAPD results and of their interpretation.

\section{RAPD of $B$. cenocepacia isolates}

Separation of fluorescently labelled RAPD products in an acrylamide gel, which was scanned by an ALFexpress II sequencer, led to clear distinction of between 10 and 25 bands over a size range of 200 to $1500 \mathrm{bp}$ for each Bcc isolate examined. Excellent reproducibility of banding patterns with only minor variations in particular band intensities was demonstrated when applied to the same samples on repeated occasions.

A total of four RAPD types were identified among the $67 \mathrm{CF}$ patients studied. Seventy-nine of the 83 isolates analysed showed a single dominant RAPD typing pattern, designated type 1 (Fig. 1). The RAPD patterns of four isolates, each recovered from a different CF patient, were distinct from the type 1 pattern and represented different types (types 2-4; type 3 was found in two of these four isolates).

One CF patient, originally infected with a unique strain, was subsequently superinfected with a strain of RAPD type 1 when examined 2 years later. The remaining 15 patients for

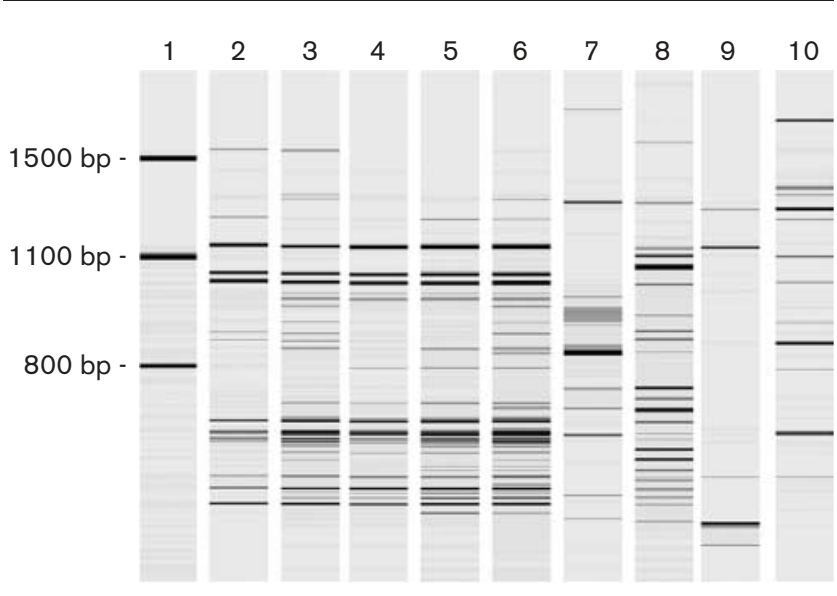

Fig. 1. RAPD typing of B. cenocepacia. RAPD-generated polymorphisms are displayed as a graphical output from the sequence analyser. Samples in each lane are as follows: 1, molecular size markers; 2 and 3, RAPD type 01 reference strains C5635 and C6965, respectively; 4-6, Czech CF patient isolates of the dominant clone CZ1; 7-9, unique Czech strains CZ2, CZ3 and CZ4, respectively; 10, ET12 reference strain C5424. 
whom serial specimens were available were all infected with strains of the same RAPD pattern (i.e. type 1) at both sampling points.

\section{PFGE of $B$. cenocepacia isolates}

Although RAPD results were indicative for the occurrence of a major B. cenocepacia transmissible strain within the Czech CF population, we sought concordance of our findings with an additional molecular typing method before drawing conclusions (Goering, 2004). Therefore, all isolates were subjected to SpeI enzyme restriction followed by PFGE, which generated bands up to $1 \mathrm{Mb}$ in size, and the fingerprints obtained were compared visually. Comparing each of the banding patterns with all the others, in total 77 out of 83 isolates yielded similar banding patterns. However, the comparison was complicated by a relatively high diversity between individual patterns, reaching up to eight fragment differences in several cases. The relative degree of similarity was validated with respect to isolates sharing banding motifs at the lower size range of macrorestriction fragments where bands were well separated (150-500 kb), and by ignoring the most frequently observed variability in larger fragments (above $600 \mathrm{~kb}$ ). When these modified criteria were applied, six out of 83 isolates had patterns significantly different from the predominant one. An illustration of PFGE fingerprint variability detected within isolates of the dominant type is shown in Fig. 2(a); dissimilarity among patterns is shown even comparing two isolates recovered in a 3-year interval from a single patient (lanes 2 and 3). Owing to a number of band differences, computer-assisted analyses predicted low similarity values and misleading clustering of isolates (data not shown).
XbaI-based macrorestriction typing was primarily performed as an additional method for 62 isolates to evaluate the significance of SpeI-digested fragment diversity. Smaller macrorestriction fragments with a maximum size of $400 \mathrm{~kb}$ were derived from this analysis. Six isolates differed in their profiles from the major XbaI-generated pulsotype, each of which corresponded to one of the six isolates of unique SpeI types detected. Significantly less variation was seen in isolates representative of the dominant RAPD type where considerable variation in SpeI fingerprints had been observed (56 out of 62 isolates examined by $\mathrm{XbaI}$; see Fig. $2 \mathrm{~b}$ ).

\section{Comparison of RAPD vs PFGE results}

The results of RAPD fingerprinting corresponded to those of PFGE for all but two clinical isolates. Seventy-seven of 83 isolates were shown to be closely related by both typing methods and designated the dominant clonal type CZ1; other strains were designated CZ2, CZ3 and CZ4, corresponding to RAPD types 2, 3 and 4, respectively. The shared consensual SpeI-generated pulsotype of CZ1 showed higher inter-pattern divergence compared to the uniform predominant RAPD pattern. Hence, the indicative value of PFGE for detecting epidemic strains conflicts with the only reported criteria for visual PFGE interpretation (Tenover et al., 1995). Nevertheless, these Tenover rules were set up for analysis of a small set of samples that were related to a single epidemic outbreak. They are not appropriate for large-scale studies (Goering, 2004; Tenover et al., 1995) when more random mutation events can be expected throughout a bacterial genome over a study period and even epidemiologically related isolates may express more pattern heterogeneity. Moreover, a higher mutation rate is likely to occur in
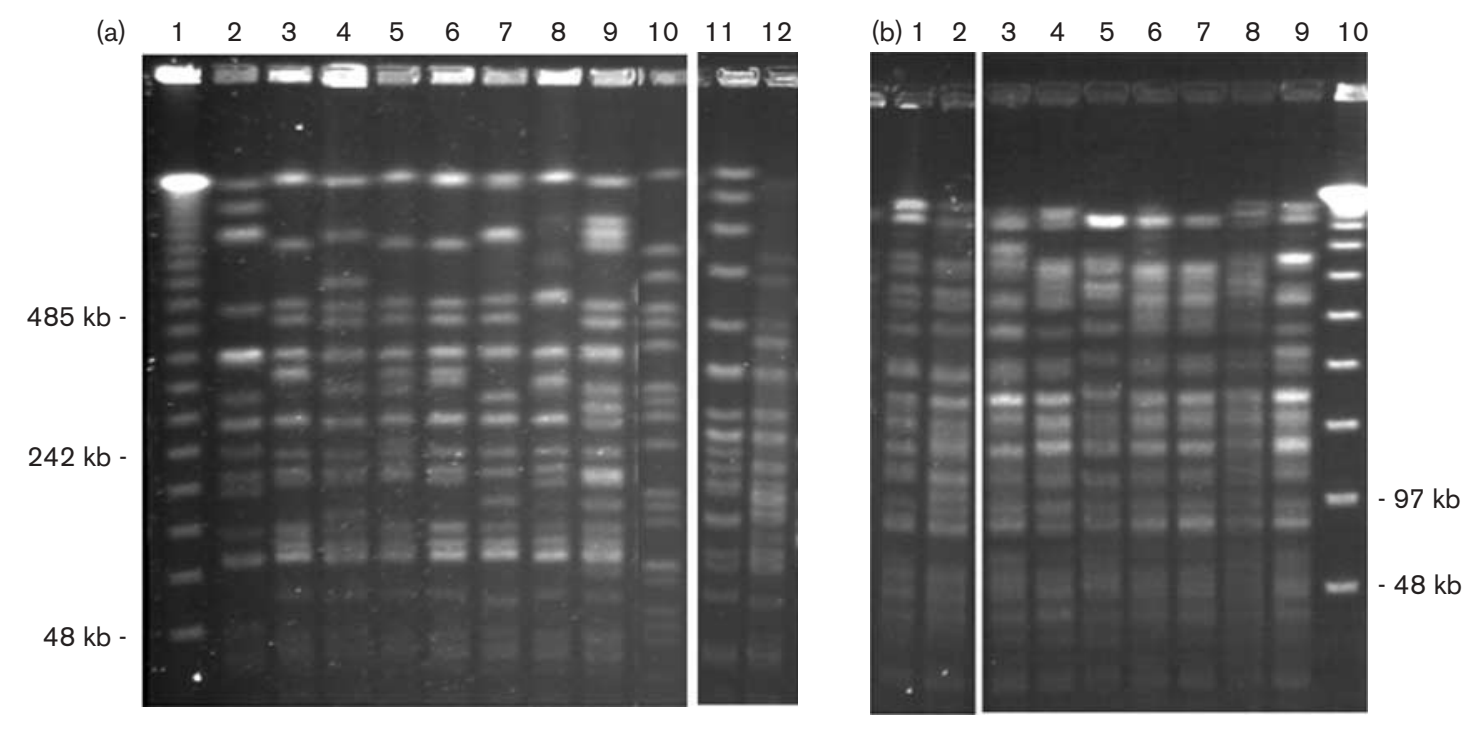

Fig. 2. PFGE of (a) Spel-digested and (b) Xbal-digested genomes of $B$. cenocepacia. (a) Samples in each lane: 1 , molecular size marker (lambda concatemer); 2-9, Czech CF patient isolates of the clone CZ1; 10, unique Czech strain CZ3; 11 and 12, RAPD type 01 reference strains C5635 and C6965, respectively. (b) Samples in each lane: 1 and 2, RAPD type 01 reference strains C5635 and C6965, respectively; $3-9$, patient isolates of the clone CZ1; 10, molecular size marker. Both parts of composite gels were matched to appropriate size using molecular size markers as a standard. 
the multireplicon genome of $B$. cenocepacia that harbours a high-copy-number of insertion-sequence elements (Lessie et al., 1996; Liu et al., 2003). Their presence in the dominant clone CZ1 may have promoted genomic rearrangements that were detected by macrorestriction with SpeI, but not observed by either XbaI analysis or RAPD typing.

Overall high concordance between PFGE and RAPD results showed that the less laborious RAPD analysis was a reliable tool for the first screening of our large isolate collection, corroborating the results observed in other studies that have used this technique to type Pseudomonas aeruginosa (Mahenthiralingam et al., 1996). In contrast, the excessively high discriminatory power of PFGE (specifically with SpeI) conferred the risk that isolates belonging to a common lineage but recovered over extended periods of time would be deemed unrelated. This oversensitivity of PFGE might be a reason for the discrepancies observed in two clinical samples, which were assigned to the unique strain group by PFGE, while RAPD analysis revealed no major differences between their patterns and the pattern of the dominant clone. Our experience with PFGE for the analysis of larger sets of $B$. cenocepacia isolates is in agreement with a comparative study of B. cenocepacia genotyping methods (Coenye et al., 2002). In this study, the authors proposed BOX-PCR fingerprinting as a more appropriate molecular tool for global epidemiological studies.

\section{cbIA and BCESM detection}

The clone CZ1, shared by multiple Czech CF patients, and the CZ4 strain were PCR positive for the auxiliary marker of transmissible B. cenocepacia strains (BCESM), but PCR negative for the genetic indicator of the ET12 strain ( $c b l A)$. Strains CZ2 and CZ3 were both BCESM- and $c b l A$-negative.

\section{Comparison of the clone CZ1 with other epidemic strains}

Based on characteristics of the other epidemic strains, we compared the Czech clone CZ1 with Canadian epidemic strains of RAPD 01, 04 and 06 types (Speert et al., 2002) as well as with the ET12 lineage, since the cblA-negative ET12 strain has been recently observed (McDowell et al., 2004). Close similarity between CZ1 and Canadian RAPD 01 was detected by both fingerprinting systems (RAPD and PFGE data are shown in Figs 1 and 2, respectively). Examination of clone CZ1 and the Canadian RAPD type as part of a Bcc multilocus sequencing typing scheme has also demonstrated that they are the same strain type (A. Baldwin, C. Dowson, E. Mahenthiralingam, unpublished data). This was a rather surprising finding. It is certainly possible that social contact between Czech and Canadian CF patients during the early 1990s may have occurred as a result of international CF camps held at that time. Attendance of CF camps has been linked to the spread of Bcc infection (Govan et al., 1993; Pegues et al., 1994); however, no direct epidemiological link between Czech and Canadian patients was found in the records available at our centre. An alternative explanation for the prevalence of a single strain type may lie in the occurrence of the strain in the proximate environment of CF patients acting as a primary source of the infection. The isolation of the epidemic PHDC strain in soil serves as a precedent for the existence of epidemic strains in natural niches (LiPuma et al., 2002).

\section{Infection control in Bcc-positive patients}

A strict isolation policy of cohorting Bcc-infected patients aimed at preventing further spread of $B$. cenocepacia infection among patients of the Prague CF Centre was introduced in 1997. However, keeping patients colonized with Bcc apart from the others has turned out to be an inadequate control measure. This measure does not reflect a risk of strain replacement with epidemic clones within the group of Bccinfected patients, which is considered an unfavourable phenomenon because a transmissible strain is likely to be more virulent than a non-transmissible one (Mahenthiralingam et al., 2001; Speert et al., 2002). As documented in this study, the epidemic clone CZ1 had replaced a unique strain in at least one patient since 1997 . We can speculate that more patients may have suffered from such superinfection events in the past, since genetic uniformity of clinical isolates throughout the Czech CF population was very high (only three out of 67 patients harboured unique strains).

It is therefore highly advisable to include the epidemicity status of isolates into rational cross-infection control guidelines and to segregate patients infected with epidemic strains of $B$. cenocepacia from those infected with sporadic unique strains. Although many CF centres practice individual segregation of patients as a means of infection control (Saiman \& Siegel, 2003), this practice is difficult in clinics where resources and space are limited. Hence, understanding the risk posed by the epidemic strains can still be useful for implementing infection control policies in centres that experience such problems. It is worth noting that identification of transmissible strains should be based on genotyping methods and not be confined to markers of transmissibility as they are not absolute markers for epidemic strains (Chen et al., 2001; McDowell et al., 2004). Lack of these markers for definitive identification was also indicated during our study where one sporadic isolate with a unique banding profile (i.e. CZ4) yielded a BCESM-positive result, but demonstrated no evidence of spread between patients.

In summary, we have identified a BCESM-positive cblAnegative epidemic clone that has been responsible for the spread of B. cenocepacia infection among Czech patients with CF. The clone, which is genetically closely related to the Canadian epidemic strain of RAPD 01 type found in multiple patients from British Columbia, Alberta and Quebec provinces, might be, in addition to the ET12 strain and the recently described $\mathrm{PHDC}$ second transatlantic clone (Coenye et al., 2004), another representative of an intercontinental epidemic lineage. Two isolates of the clone CZ1 have been deposited in the Czech Collection of Microorganisms (CCM7291 and CCM7292). 


\section{ACKNOWLEDGEMENTS}

We thank John J. LiPuma (University of Michigan, Ann Arbor, MI, USA) and Alexandr Nemec (National Institute of Public Health, Prague, Czech Republic) for helpful discussions. The excellent technical assistance of Helena Reitzova and Josef Vcelak is gratefully acknowledged. This study was supported by Ministry of Education, Czech Republic (MSM 0021620812). P. D. acknowledges Wellcome Trust for Travel Award funding his short-term visit to Cardiff School of Biosciences.

\section{REFERENCES}

Chen, J. S., Witzmann, K. A., Spilker, T., Fink, R. J. \& LiPuma, J. J. (2001). Endemicity and inter-city spread of Burkholderia cepacia genomovar III in cystic fibrosis. J Pediatr 139, 643-649.

Coenye, T., Spilker, T., Martin, A. \& LiPuma, J. J. (2002). Comparative assessment of genotyping methods for epidemiologic study of Burkholderia cepacia genomovar III. J Clin Microbiol 40, 3300-3307.

Coenye, T., Spilker, T., Van Schoor, A., LiPuma, J. J. \& Vandamme, P. (2004). Recovery of Burkholderia cenocepacia strain PHDC from cystic fibrosis patients in Europe. Thorax 59, 952-954.

Drevinek, P., Hrbackova, H., Cinek, O., Bartosova, J., Nyc, O., Nemec, A. \& Pohunek, P. (2002). Direct PCR detection of Burkholderia cepacia complex and identification of its genomovars by using sputum as source of DNA. J Clin Microbiol 40, 3485-3488.

Drevinek, P., Cinek, O., Melter, J., Langsadl, L., Navesnakova, Y. \& Vavrova, V. (2003). Genomovar distribution of the Burkholderia cepacia complex differs significantly between Czech and Slovak patients with cystic fibrosis. J Med Microbiol 52, 603-604.

Goering, R. V. (2004). Pulsed-field gel electrophoresis. In Molecular Microbiology: Diagnostic Principles and Practice, pp.185-196. Edited by D. H. Persing and others. Washington, DC: American Society for Microbiology.

Govan, J. R., Brown, P. H., Maddison, J., Doherty, C. J., Nelson, J. W., Dodd, M., Greening, A. P. \& Webb, A. K. (1993). Evidence for transmission of Pseudomonas cepacia by social contact in cystic fibrosis. Lancet 342, 15-19.

Johnson, W. M., Tyler, S. D. \& Rozee, K. R. (1994). Linkage analysis of geographic and clinical clusters in Pseudomonas cepacia infections by multilocus enzyme electrophoresis and ribotyping. J Clin Microbiol 32, 924-930.

Lessie, T. G., Hendrickson, W., Manning, B. D. \& Devereux, R. (1996). Genomic complexity and plasticity of Burkholderia cepacia. FEMS Microbiol Lett 144, 117-128.

LiPuma, J. J., Mortensen, J. E., Dasen, S. E., Edlind, T. D., Schidlow, D. V., Burns, J. L. \& Stull, T. L. (1988). Ribotype analysis of Pseudomonas cepacia from cystic fibrosis treatment centers. J Pediatr 113, 859-862.

LiPuma, J. J., Spilker, T., Coenye, T. \& Gonzalez, C. F. (2002). An epidemic Burkholderia cepacia complex strain identified in soil. Lancet 359, 2002-2003.

Liu, L., Spilker, T., Coenye, T. \& LiPuma, J. J. (2003). Identification by subtractive hybridization of a novel insertion element specific for two widespread Burkholderia cepacia genomovar III strains. J Clin Microbiol 41, 2471-2476.
Mahenthiralingam, E., Campbell, M. E., Foster, J., Lam, J. S. \& Speert, D. P. (1996). Random amplified polymorphic DNA typing of Pseudomonas aeruginosa isolates recovered from patients with cystic fibrosis. J Clin Microbiol 34, 1129-1135.

Mahenthiralingam, E., Simpson, D. A. \& Speert, D. P. (1997). Identification and characterization of a novel DNA marker associated with epidemic Burkholderia cepacia strains recovered from patients with cystic fibrosis. J Clin Microbiol 35, 808-816.

Mahenthiralingam, E., Bischof, J., Byrne, S. K., Radomski, C., Davies, J. E., Av-Gay, Y. \& Vandamme, P. (2000). DNA-based diagnostic approaches for identification of Burkholderia cepacia complex, Burkholderia vietnamiensis, Burkholderia multivorans, Burkholderia stabilis, and Burkholderia cepacia genomovars I and III. J Clin Microbiol 38, 3165-3173.

Mahenthiralingam, E., Vandamme, P., Campbell, M. E. \& 7 other authors (2001). Infection with Burkholderia cepacia complex genomovars in patients with cystic fibrosis: virulent transmissible strains of genomovar III can replace Burkholderia multivorans. Clin Infect Dis 33, $1469-1475$.

Mahenthiralingam, E., Baldwin, A. \& Vandamme, P. (2002). Burkholderia cepacia complex infection in patients with cystic fibrosis. J Med Microbiol 51, 533-538.

McDowell, A., Mahenthiralingam, E., Dunbar, K. E., Moore, J. E., Crowe, M. \& Elborn, J. S. (2004). Epidemiology of Burkholderia cepacia complex species recovered from cystic fibrosis patients: issues related to patient segregation. J Med Microbiol 53, 663-668.

Pegues, D. A., Carson, L. A., Tablan, O. C., FitzSimmons, S. C., Roman, S. B., Miller, J. M. \& Jarvis, W. R. (1994). Acquisition of Pseudomonas cepacia at summer camps for patients with cystic fibrosis. Summer Camp Study Group. J Pediatr 124, 694-702.

Saiman, L. \& Siegel, J. (2003). Infection control recommendations for patients with cystic fibrosis: microbiology, important pathogens, and infection control practices to prevent patient-to-patient transmission. Infect Control Hosp Epidemiol 24, S6-S52.

Sajjan, U. S., Sun, L., Goldstein, R. \& Forstner, J. F. (1995). Cable (cbl) type II pili of cystic fibrosis-associated Burkholderia (Pseudomonas) cepacia: nucleotide sequence of the $c b l A$ major subunit pilin gene and novel morphology of the assembled appendage fibers. J Bacteriol 177, 1030-1038.

Speert, D. P., Henry, D., Vandamme, P., Corey, M. \& Mahenthiralingam, E. (2002). Epidemiology of Burkholderia cepacia complex in patients with cystic fibrosis, Canada. Emerg Infect Dis 8, 181-187.

Sun, L., Jiang, R. Z., Steinbach, S. \& 7 other authors (1995). The emergence of a highly transmissible lineage of cbl+ Pseudomonas (Burkholderia) cepacia causing CF centre epidemics in North America and Britain. Nat Med 1, 661-666.

Tenover, F. C., Arbeit, R. D., Goering, R. V., Mickelsen, P. A., Murray, B. E., Persing, D. H. \& Swaminathan, B. (1995). Interpreting chromosomal DNA restriction patterns produced by pulsed-field gel electrophoresis: criteria for bacterial strain typing. J Clin Microbiol 33, 22332239.

Vandamme, P., Holmes, B., Coenye, T., Goris, J., Mahenthiralingam, E., LiPuma, J. J. \& Govan, J. R. (2003). Burkholderia cenocepacia sp. nov. - a new twist to an old story. Res Microbiol 154, 91-96. 